\title{
WEIGHTED SPACES OF HOLOMORPHIC FUNCTIONS ON THE UPPER HALFPLANE
}

\author{
MOHAMMAD ALI ARDALANI and WOLFGANG LUSKY
}

\begin{abstract}
We discuss weighted spaces $H v(\mathrm{G})$ of holomorphic functions on the upper halfplane $\mathrm{G}$ where $v(w)=v(i \operatorname{Im} w), w \in \mathrm{G}, \lim _{t \rightarrow 0} v(i t)=0$ and $v(i t)$ is increasing in $t$. We characterize those weights $v$ with moderate growth where $H v(\mathrm{G})$ is isomorphic to $l_{\infty}$ and we show that this is never the case if $v$ is bounded.
\end{abstract}

\section{Introduction}

Let $O \subset \mathrm{C}$ be an open subset and $v: O \rightarrow[0, \infty[$ a given function. Then we consider, for $f: O \rightarrow \mathrm{C}$, the weighted sup-norm

$$
\|f\|_{v}=\sup _{z \in O}|f(z)| v(z)
$$

and the spaces

$$
H v(O)=\left\{f: O \rightarrow \text { C holomorphic }:\|f\|_{v}<\infty\right\}
$$

and

$$
H v_{0}(O)=\{f \in H v(O):|f(z)| v(z) \text { vanishes at } \infty\} .
$$

(Here $|f| v$ vanishes at $\infty$ if for any $\epsilon>0$ there is a compact subset $K \subset O$ such that $|f(z)| v(z)<\epsilon$ for all $z \in O \backslash K$.)

Assume that $\lim _{\operatorname{dist}(z, \partial O) \rightarrow 0} v(z)=0, v(z)>0$ for all $z \in O$ and $v$ is continuous. Then, for a holomorphic function $f, f \in H v(O)$ is equivalent to the growth condition $|f(z)|=O(1 / v(z))$ as $\operatorname{dist}(z, \partial O) \rightarrow 0$ while $f \in$ $H v_{0}(O)$ is equivalent to $|f(z)|=o(1 / v(z))$ as $\operatorname{dist}(z, \partial O) \rightarrow 0$.

There is a large number of publications which deal with radial weights $v$ on $\mathrm{D}=\{z \in \mathrm{C}:|z|<1\}$ where $v(z)=v(|z|), z \in \mathrm{D}$, and $v$ satisfies in addition $v(t) \leq v(s)$ if $0 \leq s \leq t<1$ and $\lim _{t \rightarrow 1} v(t)=0$. Of particular interest here are weights with moderate decay, i.e. which satisfy the condition (U) of Shields and Williams ([12], [13], [14]). (U) is equivalent to $\sup _{n \in \mathrm{N}} v\left(1-2^{-n}\right) / v(1-$ 
$\left.2^{-n-1}\right)<\infty$ (see [4]). In [10] it was shown that for such weights $H v(\mathrm{D})$ is isomorphic to $l_{\infty}$ if and only if $\inf _{k \in \mathbb{N}} \lim \sup _{n \rightarrow \infty} v\left(1-2^{-n-k}\right) / v\left(1-2^{-n}\right)<$ 1. The latter condition corresponds to condition (L) of Shields and Williams ([4], [12], [13], [14]). It turns out that even without (U) the Banach space $H v(\mathrm{D})$ is always isomorphic either to $l_{\infty}$ or to $H_{\infty}$, the space of all bounded holomorphic functions endowed with the sup-norm ([11]). Weights on $D$ which satisfy both (U) and (L) are called normal weights. They have been studied extensively.

Inspired by these results about radial weights on $D$ we consider in this paper the upper halfplane $\mathrm{G}=\{w \in \mathrm{C}: \operatorname{Im} w>0\}$ and investigate the following class of weights.

Definition 1.1. (i) Let $v$ be a continuous function on G satisfying $v(w)>$ 0 for all $w \in \mathrm{G}$. Assume that $v$ satisfies

$$
\lim _{r \rightarrow 0} v(\text { ir })=0 \text { and } v\left(w_{1}\right) \leq v\left(w_{2}\right) \quad \text { whenever } 0<\operatorname{Im}\left(w_{1}\right) \leq \operatorname{Im}\left(w_{2}\right) .
$$

Then $v$ is called a standard weight.

(ii) A standard weight $v$ on G satisfies condition ( $\star$ ) if

$$
\sup _{k \in \mathbf{Z}} \frac{v\left(2^{k+1} i\right)}{v\left(2^{k} i\right)}<\infty .
$$

A standard weight always satisfies $v(w)=v(i \operatorname{Im} w)$ for all $w \in \mathrm{G}$ which is a consequence of the definition.

In contrast to radial weights on $D$ very little is known about standard weights $v$ on $\mathrm{G}$. We mention Stanev's result ([15]) that there exists some $b \in \mathrm{R}$ with $v(i t) \leq e^{b t}, t>0$, if and only if $H v(\mathrm{G}) \neq\{0\}$. This is always the case if $(\star)$ holds. $H v_{0}(\mathrm{G})$ is always isomorphic to a subspace of $c_{0}$ ([3]). Moreover, if $v$ is a bounded standard weight on $\mathrm{G}$ then $H v_{0}(\mathrm{G})$ has a Schauder basis ([1]). Finally, with the methods of [2] one can show that $H v_{0}(\mathrm{G})^{\star \star}$ is isometrically isomorphic to $H v(\mathrm{G})$ (see [5]). (The results of [1], [3], [5], [15] even hold for a larger class of weights.)

In our paper we want to contribute to the isomorphic classification of $H v(\mathrm{G})$ and $H v_{0}(\mathrm{G})$. We show

THEOREM 1.2. Let $v$ be a standard weight on $\mathrm{G}$ satisfying ( $\star)$. Then the following are equivalent

(i) $H v(\mathrm{G})$ is isomorphic to $l_{\infty}$

(ii) $H v_{0}(\mathrm{G})$ is isomorphic to $c_{0}$ 
(iii) $v$ also satisfies $(\star \star)$ :

$$
\inf _{n \in \mathrm{N}} \sup _{k \in \mathbf{Z}} \frac{v\left(2^{k} i\right)}{v\left(2^{k+n} i\right)}<1 .
$$

ExAmPle. Let $\beta>0>\gamma$ and put

$$
\begin{aligned}
& v_{1}(w)=(\operatorname{Im}(w))^{\beta}, \\
& v_{2}(w)=\min \left(v_{1}(w), 1\right), \\
& v_{3}(w)= \begin{cases}(1-\log (\operatorname{Im}(w)))^{\gamma} & \text { if } \operatorname{Im}(w) \leq 1 \\
\operatorname{Im} w & \text { if } \operatorname{Im}(w)>1 .\end{cases}
\end{aligned}
$$

All these weights are standard weights. $v_{1}$ satisfies $(\star)$ and $(\star \star)$ while $v_{2}$ and $v_{3}$ satisfy only $(\star)$.

We immediately get:

COROLLARY 1.3. If $v$ is a bounded standard weight on $\mathrm{G}$ satisfying $(\star)$ then $H v(\mathrm{G})$ is never isomorphic to $l_{\infty}$.

The conditions $(\star)$ and $(\star \star)$ resemble the conditions for normal radial weights $u$ on D, see [4], [10], [12], [13], [14] and Lemma 1.6 below. However if we consider a Möbius transform $\alpha: \mathrm{D} \rightarrow \mathrm{G}$ then $v \circ \alpha$ is non-radial on $\mathrm{D}$ and we do not have $\lim _{|z| \rightarrow 1}(v \circ \alpha)(z)=0$. Therefore it is not possible to derive Theorem 1.2 directly from the corresponding results of radial weights on $\mathrm{D}$.

The main ingredients of the proof of Theorem 1.2 are the following

Proposition 1.4. Let $v$ be a standard weight on $\mathrm{G}$ and put

$$
\begin{aligned}
& v_{n}(w)=v\left(\frac{4 \operatorname{Im} w}{\left(\left|\frac{w}{n}+i\right|+\left|\frac{w}{n}-i\right|\right)^{2}} i\right), \quad w \in \mathrm{G}, \\
& u_{n}(z)=v\left(n \frac{1-|z|}{1+|z|} i\right), \quad z \in \mathrm{D}, \quad n \in \mathrm{N} .
\end{aligned}
$$

Then $v_{n}(w) \uparrow v(w), w \in \mathrm{G}$, and $H v_{n}(\mathrm{G})$ is isometrically isomorphic to $H u_{n}(\mathrm{D})$. Moreover, $u_{n}$ is a radial weight on $\mathrm{D}$ such that $u_{n}(t)$ is decreasing in $t \in[0,1[$.

Proof. We only have to show that $H v_{n}(\mathrm{G})$ and $H u_{n}(\mathrm{D})$ are isometrically isomorphic. To this end consider $\alpha_{n}: \mathrm{D} \rightarrow \mathrm{G}$ with $\alpha_{n}(z)=n(1+z)(1-z)^{-1} i$. Then $\alpha_{n}^{-1}(w)=(w / n-i)(w / n+i)^{-1}, w \in \mathrm{G}$. We have $v_{n} \circ \alpha_{n}=u_{n}$. Hence 
$T: H v_{n}(\mathrm{G}) \rightarrow H u_{n}(\mathrm{D})$ with $(T f)(z)=f\left(\alpha_{n}(z)\right), z \in \mathrm{D}, f \in H v_{n}(\mathrm{G})$, is an onto-isometry.

Corollary 1.5. Let $v$ be a standard weight on $\mathrm{G}$ and let $u_{n}$ be the weights on $\mathrm{D}$ of Proposition 1.4. Then $\mathrm{Hv}(\mathrm{G})$ is isometrically isomorphic to a complemented subspace of $\left(\sum_{n=1}^{\infty} \oplus H u_{n}(\mathrm{D})\right)_{(\infty)}$.

Proof. In view of Proposition 1.4 it suffices to show that $H v(\mathrm{G})$ is isometrically isomorphic to a complemented subspace of $\left(\sum_{n=1}^{\infty} \oplus H v_{n}(\mathrm{G})\right)_{(\infty)}$. To this end define $T: H v(\mathrm{G}) \rightarrow\left(\sum_{n=1}^{\infty} \oplus H v_{n}(\mathrm{G})\right)_{(\infty)}$ by $T f=(f, f, \ldots) . T$ is an isometry since $v_{n} \uparrow v$.

Now, let $\left(f_{n}\right) \in\left(\sum_{n=1}^{\infty} \oplus H v_{n}(\mathrm{G})\right)_{(\infty)}$. If $K \subset \mathrm{G}$ is compact then $\inf _{w \in K} \operatorname{Im} w>0$ and hence $c:=\inf _{n \in \mathrm{N}} \inf _{w \in K} v_{n}(w) \geq \inf _{w \in K} v_{1}(w)>0$. This implies $\sup _{n \in \mathrm{N}} \sup _{w \in K}\left|f_{n}(w)\right| \leq c^{-1} \sup _{n}\left\|f_{n}\right\|_{v_{n}}$. Fix a free ultrafilter $\mathscr{U}$ on $\mathrm{N}$ and put $\left(S\left(f_{n}\right)\right)(w)=\lim _{n, \mathscr{U}} f_{n}(w)$. By Montel's theorem $S\left(f_{n}\right)$ is holomorphic. We have $\left\|S\left(f_{n}\right)\right\|_{v} \leq \sup _{n}\left\|f_{n}\right\|_{v_{n}}$ in view of Proposition 1.4. Clearly $T S$ is a contractive projection from $\left(\sum_{n=1}^{\infty} \oplus H v_{n}(\mathrm{G})\right)_{(\infty)}$ onto $T H v(\mathrm{G})$.

We complete the proof of Theorem 1.2 in Section 4. Before, in Section 2, we discuss the space $H u(\mathrm{D})$ for a radial weight $u$ on $\mathrm{D}$ and we consider special subspaces of $H v(\mathrm{G})$ in Section 3.

Here we prove

Lemma 1.6. Let $v$ be a standard weight on $\mathrm{G}$. Then

(i) $a:=\sup _{k \in \mathrm{Z}} \frac{v\left(2^{k+1} i\right)}{v\left(2^{k} i\right)}<\infty$ if and only if $\frac{v(t i)}{v(s i)} \leq c\left(\frac{t}{s}\right)^{\beta}$ whenever $0<$ $s \leq t$, for some $c>0$ and $\beta>0$. In this case we can take $c=a^{2}$ and $\beta=\frac{\log a}{\log 2}$.

(ii) $\inf _{n \in \mathrm{N}} \sup _{k \in \mathrm{Z}} \frac{v\left(2^{k} i\right)}{v\left(2^{k+n} i\right)}<1$ if and only if $\frac{v(t i)}{v(s i)} \geq d\left(\frac{t}{s}\right)^{\gamma}$ whenever $0<s \leq$ $t$, for some constants $d, \gamma>0$.

Proof. (i) Assume $a<\infty$. Put $\beta=\log a / \log 2$. Then fix $s, t$ with $2^{k} \leq$ $s \leq 2^{k+1}$ and $2^{n+k} \leq t \leq 2^{n+k+1}$ for some $n \in \mathrm{N} \cup\{0\}$ and $k \in \mathrm{Z}$. We obtain

$$
\frac{v(t i)}{v(s i)} \leq a^{n+1}=\left(2^{n+1}\right)^{\beta}=\left(\frac{2^{n+k+2}}{2^{k+1}}\right)^{\beta} \leq 2^{2 \beta}\left(\frac{t}{s}\right)^{\beta}=a^{2}\left(\frac{t}{s}\right)^{\beta} .
$$

If $v(t i) / v(s i) \leq c(t / s)^{\beta}$ whenever $0<s \leq t$ then put $t=2^{k+1}$ and $s=2^{k}$. This yields $v\left(2^{k+1} i\right) / v\left(2^{k} i\right) \leq c 2^{\beta}$.

(ii) Assume there is $n \in \mathrm{N}$ and $b \in] 0,1[$ with

$$
\frac{v\left(2^{k} i\right)}{v\left(2^{k+n} i\right)} \leq b \quad \text { for all } \quad k \in \mathrm{Z}
$$


We may take $b \leq 1 / 2$. Otherwise consider $m n$ instead of $n$ for suitable $m \in \mathrm{N}$.

Fix $0<s \leq t$. Then there is $k \in \mathbf{Z}, l \in \mathrm{N} \cup\{0\}$ with $2^{k n} \leq s \leq 2^{(k+1) n}$ and $2^{(l+k) n} \leq t \leq 2^{(l+k+1) n}$. Assume $l>1$. Then we have

$$
\frac{v(s i)}{v(t i)} \leq \frac{v\left(2^{(k+1) n} i\right)}{v\left(2^{(l+k) n} i\right)} \leq\left(\frac{1}{2}\right)^{l-1}=2^{2}\left(\frac{2^{k n}}{2^{(l+k+1) n}}\right)^{1 / n} \leq 2^{2}\left(\frac{s}{t}\right)^{1 / n} .
$$

If $l \leq 1$ then

$$
\frac{v(s i)}{v(t i)} \leq \frac{v\left(2^{(k+1) n} i\right)}{v\left(2^{(l+k) n} i\right)} \leq a \leq 2^{2} a\left(\frac{2^{k n}}{2^{(l+k+1) n}}\right)^{1 / n} \leq 2^{2} a\left(\frac{s}{t}\right)^{1 / n} .
$$

Put $\gamma=1 / n$ and $d=1 /(4 a)$.

If

$$
\frac{v(t i)}{v(s i)} \geq d\left(\frac{t}{s}\right)^{\gamma} \quad \text { for } \quad 0<s \leq t
$$

then take $n \in \mathrm{N}$ such that $d 2^{-n \gamma} \leq 2^{-1}$. With $s=2^{k}, t=2^{k+n}$ we obtain

$$
\frac{v\left(2^{k} i\right)}{v\left(2^{k+n} i\right)} \leq d \frac{1}{2^{n \gamma}} \leq \frac{1}{2} \quad \text { for all } \quad k \in \mathbf{Z}
$$

For two Banach spaces $X, Y$ put

$$
d(X, Y)=\inf \left\{\|T\| \cdot\left\|T^{-1}\right\|: T: X \rightarrow Y \text { an onto-isomorphism }\right\}
$$

provided $X$ and $Y$ are isomorphic (otherwise put $d(X, Y)=\infty) . d(X, Y)$ is called the Banach-Mazur distance between $X$ and $Y$ ([17]).

If $X \subset Y$ we define

$$
\lambda(X, Y)=\inf \{\|P\|: P: Y \rightarrow X \text { a projection }\}
$$

and $\lambda(X)=\sup _{Y \supset X} \lambda(X, Y) \cdot \lambda(X, Y)$ and $\lambda(X)$ are called the relative and absolute projection constant of $X$ ([17]). We have $\lambda(X) \leq d\left(X, l_{\infty}\right)$ and $\lambda(X)=\lambda\left(X, l_{\infty}\right)$ if $X \subset l_{\infty}$. This follows from the Hahn-Banach extension property of $l_{\infty}$ which also shows $\lambda\left(l_{\infty}\right)=1$. Moreover, using the same argument, we can replace $l_{\infty}$ by $L_{\infty}$. If $Y$ is another Banach space then $\lambda(X) \leq \lambda(Y) d(X, Y)$ ([17]). Finally, if $\operatorname{dim} X=n$ it is easily seen that $\lambda(X) \leq n$. (We even have $\lambda(X) \leq \sqrt{n},[7]$.)

\section{Radial weights on $D$}

Let $R>0$. For a function $f: R \cdot \mathrm{D} \rightarrow \mathrm{C}$ and $0 \leq r<R$ put $M_{\infty}(f, r)=$ $\sup _{|z|=r}|f(z)|$. Using the maximum principle we obtain (e.g., see [11], Lemma 3.1) 
LEMMA 2.1. Let $0<r<s$.

(i) If $f$ is a polynomial of degree $n$ then

$$
M_{\infty}(f, s) \leq\left(\frac{s}{r}\right)^{n} M_{\infty}(f, r) .
$$

(ii) If $g(z)=\sum_{k=m}^{n} \alpha_{k} z^{k}$ then

$$
M_{\infty}(g, r) \leq\left(\frac{r}{s}\right)^{m} M_{\infty}(g, s) .
$$

Now let $u$ be a radial weight on $\mathrm{D}$ such that $u(t)$ is decreasing in $t \in[0,1[$ and $\lim _{t \rightarrow 1} u(t)=0$. Assume

$$
a:=\sup _{n \in \mathrm{N}} \frac{u\left(1-1 / 2^{n}\right)}{u\left(1-1 / 2^{n+1}\right)}<\infty .
$$

Using induction we find integers $m_{0}=0<m_{1}<m_{2}<m_{3}<\cdots$ such that

$$
\frac{1}{2 a} \leq \frac{u\left(1-1 / 2^{m_{k+1}}\right)}{u\left(1-1 / 2^{m_{k}}\right)} \leq \frac{1}{2}
$$

(e.g., let $m_{k+1}$ be the smallest integer with $u\left(1-2^{-m_{k+1}}\right) / u\left(1-2^{-m_{k}}\right) \leq 1 / 2$ ).

For a harmonic function $f\left(r e^{i \varphi}\right)=\sum_{k \in Z} \alpha_{k} r^{|k|} e^{i k \varphi}$ and $n \in \mathrm{N}$ put

$$
\left(R_{n} f\right)\left(r e^{i \varphi}\right)=\sum_{|k| \leq 2^{n}} \alpha_{k} r^{|k|} e^{i k \varphi}+\sum_{2^{n}<|k|<2^{n+1}} \alpha_{k} \frac{2^{n+1}-|k|}{2^{n}} r^{|k|} e^{i k \varphi} .
$$

Then we obtain

LEMMA 2.2.

(i) $R_{n} R_{m}=R_{\min (m, n)}$ if $n \neq m$.

(ii) $M_{\infty}\left(R_{n} f, r\right) \leq 3 M_{\infty}(f, r)$ for any $n \in \mathrm{N}$ and any $r>0$.

Proof. (i) follows from the definition. (ii) follows, e.g., from [11], Lemma 3.3.

We need a slightly stronger result than Theorem (i) (d) and (ii) (c) for holomorphic functions in [10].

Proposition 2.3. Put $\|f\| \|=\sup _{k} M_{\infty}\left(\left(R_{m_{k}}-R_{m_{k-1}}\right) f, 1\right) u\left(1-2^{-m_{k}}\right)$. There is a universal constant $b>0$, depending only on $a$, such that

$$
\frac{1}{96}\|f \mid\| \leq\|f\|_{u} \leq b\|f\| \| \quad \text { for any } \quad f \in H u(\mathrm{D}) .
$$


Proof. Fix $0<r<1$, say $1-2^{-m_{k-1}} \leq r \leq 1-2^{-m_{k}}$. Put $f_{j}=$ $\left(R_{m_{j}}-R_{m_{j-1}}\right) f$ (where $R_{m_{-1}}=0$ ) and $r_{j}=1-2^{-m_{j}}, j=0,1,2, \ldots$ Using Lemma 2.1 and (2.1) we obtain, for $j \leq k$,

$$
\begin{aligned}
M_{\infty}\left(f_{j}, r\right) u(r) & \leq 2 a M_{\infty}\left(f_{j}, r_{k}\right) u\left(r_{k}\right) \\
& \leq 2 a\left(\frac{r_{k}}{r_{j}}\right)^{2^{m_{j}+1}} \frac{u\left(r_{k}\right)}{u\left(r_{j}\right)} M_{\infty}\left(f_{j}, r_{j}\right) u\left(r_{j}\right) \\
& \leq 2 a r_{j}^{-2^{m_{j}+1}} 2^{j-k} M_{\infty}\left(f_{j}, 1\right) u\left(r_{j}\right) .
\end{aligned}
$$

For $l \geq k$ we have

$$
\begin{aligned}
M_{\infty}\left(f_{l}, r\right) u(r) & \leq 2 a M_{\infty}\left(f_{l}, r_{k}\right) u\left(r_{k}\right) \\
& \leq 2 a\left(\frac{r_{k}}{r_{l}}\right)^{2^{m_{l}-1}} \frac{u\left(r_{k}\right)}{u\left(r_{l}\right)} M_{\infty}\left(f_{l}, r_{l}\right) u\left(r_{l}\right) \\
& \leq 2 a\left(\frac{r_{k}}{r_{l}}\right)^{2^{m_{l}-1}}(2 a)^{l-k} M_{\infty}\left(f_{l}, 1\right) u\left(r_{l}\right)
\end{aligned}
$$

Put

$$
b_{k}=2 a\left(\sum_{j=1}^{k-1} r_{j}^{-2^{m_{j}+1}} 2^{j-k}+\sum_{l=k+1}^{\infty}\left(\frac{r_{k}}{r_{l}}\right)^{2^{m_{l-1}}}(2 a)^{l-k}\right) .
$$

Then, using the Bernoulli inequality and $1-x \leq e^{-x}$ for $x \geq 0$, we obtain

$$
\begin{aligned}
b_{k} & \leq 2 a\left(\sum_{j=1}^{k-1} \frac{16}{2^{k-j}}+\sum_{l=k+1}^{\infty} 2 \exp \left(-2^{m_{l-1}-m_{k}}+(\log 2+\log a)(l-k)\right)\right) \\
& \leq 32 a+4 a \sum_{l=k+1}^{\infty} \exp \left(-2^{l-k-1}+(l-k)(\log 2+\log a)\right) .
\end{aligned}
$$

We see that there is $b>0$ depending only on $a$ with $b_{k} \leq b$ for all $k$. Since $f$ as holomorphic function on $\mathrm{D}$ has a Taylor series which converges uniformly on $r \overline{\mathrm{D}}$ we obtain that $f=\sum_{j=1}^{\infty} f_{j}$ and the series converges uniformly on $r \overline{\mathrm{D}}$. Hence

$$
M_{\infty}(f, r) u(r) \leq \sum_{j=1}^{\infty} M_{\infty}\left(f_{j}, r\right) u(r) \leq b \sup _{j} M_{\infty}\left(f_{j}, 1\right) u\left(r_{j}\right)
$$


which implies $\|f\|_{u} \leq b\|f \mid\|$. The lower estimate follows from

$$
\begin{aligned}
M_{\infty}\left(f_{j}, 1\right) u\left(r_{j}\right) & \leq r_{j}^{-2^{m_{j}+1}} M_{\infty}\left(f_{j}, r_{j}\right) u\left(r_{j}\right) \\
& \leq 16 \cdot 6 M_{\infty}\left(f, r_{j}\right) u\left(r_{j}\right) \\
& \leq 96\|f\|_{u} .
\end{aligned}
$$

For a harmonic function $f\left(r e^{i \varphi}\right)=\sum_{k \in \mathrm{Z}} \alpha_{k} r^{|k|} e^{i k \varphi}$ put $(R f)(z)=$ $\sum_{k=0}^{\infty} \alpha_{k} z^{k}$.

Lemma 2.4. For any $m, n \in \mathrm{N}$ with $m \leq n$, any trigonometric polynomial of the form $f\left(r e^{i \varphi}\right)=\sum_{m<|k| \leq n} \alpha_{k} r^{|k|} e^{i k \varphi}$ and any $r>0$ we have

$$
M_{\infty}(R f, r) \leq \frac{n}{m} M_{\infty}(f, r) .
$$

Proof. See [11], Lemma 3.3(b).

Proposition 2.5. There are universal constants $c_{1}, c_{2}, c_{3}>0$, depending only on a, such that for any sequence $\left(m_{k}\right)$ with (2.1) we have

$$
c_{1} \sup _{k}\left(m_{k}-m_{k-1}\right) \leq \lambda(H u(\mathrm{D})) \leq c_{2} \sup _{k} 2^{m_{k}-m_{k-1}} .
$$

Moreover, there is an (into-)isomorphism $T: H u(\mathrm{D}) \rightarrow l_{\infty}$ with $\|T\| \cdot$ $\left\|T^{-1}\right\| \leq c_{3}$.

Proof. To prove the left-hand inequality we can assume $\lambda(H u(\mathrm{D}))<\infty$. Put $h u(\mathrm{D})=\left\{f: \mathrm{D} \rightarrow \mathrm{C}\right.$ harmonic $\left.:\|f\|_{u}<\infty\right\}$. Fix $\epsilon>0$ and find a projection $P: h u(\mathrm{D}) \rightarrow H u(\mathrm{D})$ with $\|P\| \leq(1+\epsilon) \lambda(H u(\mathrm{D}))$. For $|\theta|=1$ put $\left(L_{\theta} f\right)(z)=f(\theta z)$. Then

$$
(R f)(z)=\frac{1}{2 \pi} \int_{0}^{2 \pi}\left(L_{e^{-i \varphi}} P L_{e^{i \varphi}} f\right)(z) d \varphi
$$

(check the Fourier series of $f$ and $P f$ ). Hence $\|R\| \leq\|P\|$. This implies $\|R\| \leq \lambda(H u(\mathrm{D}))$.

Consider $f(z)=\sum_{k=1}^{\infty} k^{-1}\left(z^{k}-\bar{z}^{k}\right) . f\left(e^{i \varphi}\right)$ is the Fourier series of the function $i(\pi-\varphi), \varphi \in[0,2 \pi]$. Hence $M_{\infty}(f, 1) \leq \pi$. Fix $k$ and assume $m_{k}-m_{k-1}>3$. Put

$$
g=\frac{\left(R_{m_{k}-1}-R_{m_{k-1}+1}\right) f}{u\left(1-2^{-m_{k}}\right)} .
$$


With the norm $|\|\cdot\|| \mid$ of Proposition 2.3, since

$$
\left(R_{m_{j}}-R_{m_{j-1}}\right)\left(R_{m_{k}-1}-R_{m_{k-1}+1}\right)=\left\{\begin{array}{ll}
0, & j \neq k \\
R_{m_{k}-1}-R_{m_{k-1}+1}, & j=k
\end{array},\right.
$$

we conclude

$$
\|g\|=M_{\infty}(g, 1) \leq 6 M_{\infty}(f, 1) \leq 6 \pi .
$$

Hence $\|g\|_{u} \leq 6 b \pi$. On the other hand,

$$
\begin{aligned}
\|R g\|_{u} & \geq \frac{1}{96} M_{\infty}(R g, 1) u\left(1-\frac{1}{2^{m_{k}}}\right) \\
& \geq \frac{1}{96}\left(\left(R_{m_{k}-1}-R_{m_{k-1}+1}\right) R f\right)(1) \\
& \geq \frac{1}{96} \sum_{j=2^{m_{k-1}+2}}^{2^{m_{k}-1}} \frac{1}{j} \\
& \geq \frac{1}{96}(\log 2)\left(m_{k}-m_{k-1}-3\right) .
\end{aligned}
$$

(Here we used $(R g) u\left(1-2^{-m_{k}}\right)=\left(R_{m_{k}-1}-R_{m_{k-1}+1}\right) R f$.) This implies

$$
\begin{aligned}
\frac{\log 2}{96}\left(m_{k}-m_{k-1}-3\right) & \leq\|R\| \cdot\|g\|_{u} \\
& \leq 6 \pi b \lambda(H u(\mathrm{D})) .
\end{aligned}
$$

If $m_{k}-m_{k-1} \leq 3$ then certainly $m_{k}-m_{k-1} \leq 3 \lambda(H u(\mathrm{D}))$. Altogether we conclude

$$
\sup _{k}\left(m_{k}-m_{k-1}\right) \leq\left(\frac{96}{\log 2} 6 \pi b+3\right) \lambda(H u(\mathrm{D})) .
$$

For the right-hand inequality put $\|f\|_{k}=M_{\infty}(f, 1) u\left(1-2^{-m_{k}}\right)$. Then $X_{k}:=$ $\left(L_{\infty}(\partial \mathrm{D}),\|\cdot\|_{k}\right)$ is isometric to $L_{\infty}$. Put $X=\left(\sum_{k} \oplus X_{k}\right)_{(\infty)}$. We have $d\left(X, l_{\infty}\right)$ $<\infty$ since $d\left(L_{\infty}, l_{\infty}\right)<\infty$. Define $T: H u(\mathrm{D}) \rightarrow X$ by $T f=\left(\left(R_{m_{k}}-\right.\right.$ $\left.\left.R_{m_{k-1}}\right) f\right)$. Then $\|T\| \leq 96$ and $\left\|T^{-1}\right\| \leq b$ in view of Proposition 2.3. Define $S: X \rightarrow H u(\mathrm{D})$ by $S\left(g_{k}\right)=\sum_{k=1}^{\infty} R\left(R_{m_{k}}-R_{m_{k-1}}\right) g_{k}$ (where the polynomial $R\left(R_{m_{k}}-R_{m_{k-1}}\right) g_{k}$ defined on $\partial \mathrm{D}$ is extended naturally to $\left.\mathrm{D}\right)$. We obtain with 
Lemma 2.4 and (2.1)

$$
\begin{aligned}
\left\|S\left(g_{k}\right)\right\|_{u} \leq & b\left\|S\left(g_{k}\right)\right\| \\
\leq & b \sup _{k}\left(2^{m_{k}-m_{k-1}} M_{\infty}\left(\left(R_{m_{k}}-R_{m_{k-1}}\right) g_{k}, 1\right)\right. \\
& \left.\quad \cdot \max \left(u\left(1-\frac{1}{2^{m_{k-1}}}\right), u\left(1-\frac{1}{2^{m_{k}}}\right), u\left(1-\frac{1}{2^{m_{k+1}}}\right)\right)\right) \\
\leq & 6 a b \sup _{k} 2^{m_{k}-m_{k-1}} \sup _{k}\left\|g_{k}\right\|_{k} .
\end{aligned}
$$

Here we used

$$
\left(R_{m_{j}}-R_{m_{j-1}}\right)\left(R_{m_{k}}-R_{m_{k-1}}\right)=0 \quad \text { if } \quad j \neq k-1, k, k+1
$$

(see Lemma 2.2(i)).

We have $T S T=T$ which is a consequence of the definition of $S$ and $T$. Hence $T S$ is a projection from $X$ onto $T H u(\mathrm{D})$. We conclude

$$
\begin{aligned}
\lambda(H u(\mathrm{D})) & \leq 96 b \lambda(T H u(\mathrm{D})) \\
& \leq 96 b d\left(L_{\infty}, l_{\infty}\right) \lambda(T H u(\mathrm{D}), X) \\
& \leq 96 b d\left(L_{\infty}, l_{\infty}\right)\|T S\| \\
& \leq 6 \cdot 96 a b^{2} d\left(L_{\infty}, l_{\infty}\right) \sup _{k} 2^{m_{k}-m_{k-1}} .
\end{aligned}
$$

\section{A special subspace of $H v(G)$}

Let $v$ be a standard weight on $\mathrm{G}$ and assume there are constants $c>0$ and $\beta>0$ with

$$
\frac{v(t i)}{v(s i)} \leq c\left(\frac{t}{s}\right)^{\beta} \quad \text { whenever } \quad 0<s \leq t .
$$

By perhaps increasing $\beta$ we may assume that $\beta$ is an even integer. We consider the subspace

$$
U_{v}=\left\{f \in H v(\mathbf{G}): w^{2 \beta} f(w)=f\left(-\frac{1}{w}\right), w \in \mathbf{G}\right\} .
$$

Note that any $f \in H v(\mathrm{G})$ has a representation of the form

$$
f(w)=\sum_{k=0}^{\infty} \alpha_{k} \frac{1}{(w+i)^{2 \beta}}\left(\frac{w-i}{w+i}\right)^{k}
$$


where the series converges uniformly on compact subsets of G. (Apply the Möbius transform $\alpha(z)=(1+z)(1-z)^{-1} i$ where $\alpha^{-1}(w)=(w-i)(w+i)^{-1}$. The function $2^{2 \beta}(1-z)^{-2 \beta} f(\alpha(z))$ is holomorphic on D. Hence $f(\alpha(z))=$ $\sum_{k=0}^{\infty} \alpha_{k} 2^{-2 \beta}(1-z)^{2 \beta} z^{k}$ for some $\alpha_{k}$ which yields the above representation.)

It can be shown that $U_{v}$ consists of the functions $f \in H v(\mathrm{G})$ with

$$
f(w)=\sum_{k=0}^{\infty} \alpha_{k} \frac{1}{(w+i)^{2 \beta}}\left(\frac{w-i}{w+i}\right)^{2 k}
$$

for some $\alpha_{k}$. For example, it is easily seen that $(w+i)^{-2 \beta}(w-i)^{2 k}(w+i)^{-2 k} \in$ $U_{v}$ for all $k \in \mathrm{N} \cup\{0\}$ (in view of (3.1)).

Proposition 3.1. Let

$$
u(z)=v\left(\frac{1-|z|}{1+|z|} i\right), \quad z \in \mathrm{D} .
$$

Then $d\left(U_{v}, H u(\mathrm{D})\right) \leq 2^{3 \beta} c^{3}$ where $c$ is the constant in (3.1).

Proof. Put $\tilde{v}(w)=(w+i)^{-2 \beta} v(w)$. Then

$$
\frac{\tilde{v}(w)}{\tilde{v}(-1 / w)}=\frac{v(w)}{|w|^{2 \beta} v(-1 / w)} \leq c \quad \text { if } \quad|w| \geq 1
$$

in view of (3.1). Define $T: U_{v} \rightarrow H \tilde{v}(\mathrm{G})$ by $(T f)(w)=(w+i)^{2 \beta} f(w)$. Then $T$ is an isometry onto $\{g \in H \tilde{v}(\mathrm{G}): g(w)=g(-1 / w), w \in \mathrm{G}\}$. For any $g \in$ $T U_{v}$ and $w \in \mathrm{G}$ with $|w| \geq 1$ we have $|g(w)| \tilde{v}(w) \leq|g(-1 / w)| \tilde{v}(-1 / w) c$. Hence

$$
\|g\|_{\tilde{v}} \leq c \sup _{w \in \mathbf{G},|w| \leq 1}|g(w)| \tilde{v}(w) .
$$

We use the Möbius transform $\alpha: \mathrm{D} \rightarrow \mathrm{G}$ with $\alpha(z)=(1+z)(1-z)^{-1} i$. Here $|\alpha(z)| \leq 1$ is equivalent to $\operatorname{Re} z \leq 0$. We have, for $z \in \mathrm{D}$,

$$
\operatorname{Im} \alpha(-|z|)=\frac{1-|z|}{1+|z|} \leq \frac{1-|z|^{2}}{|1-z|^{2}}=\operatorname{Im} \alpha(z) .
$$

Hence,

$$
\frac{1}{c} \tilde{v}(\alpha(z)) \leq v(\alpha(-|z|))=u(z) \leq 2^{\beta} \tilde{v}(\alpha(z)) \quad \text { if } \quad \operatorname{Re} z \leq 0 .
$$

Indeed, in view of (3.1),

$$
\frac{\tilde{v}(\alpha(z))}{v(\alpha(-|z|))} \leq c\left(\frac{1-|z|^{2}}{|1-z|^{2}}\right)^{\beta}\left(\frac{1+|z|}{1-|z|}\right)^{\beta} \frac{|1-z|^{2 \beta}}{2^{2 \beta}} \leq c .
$$


On the other hand,

$$
v(\alpha(-|z|)) \leq v(\alpha(z))=\tilde{v}(\alpha(z)) \frac{2^{2 \beta}}{|1-z|^{2 \beta}} \leq \tilde{v}(\alpha(z)) 2^{\beta}
$$

since $\operatorname{Re} z \leq 0$. This shows (3.3).

Put $X=\{h \in H u(\mathrm{D}): h(z)=h(-z), z \in \mathrm{D}\}$. (3.2) and (3.3) imply

$$
d\left(U_{v}, X\right) \leq 2^{\beta} c^{2} .
$$

Now, for $h \in H u(\mathrm{D})$ let $(S h)(z)=h\left(z^{2}\right)$. Then, by (3.1),

$$
|(S h)(z)| u(z)=\left|h\left(z^{2}\right)\right| v\left(\alpha\left(-|z|^{2}\right)\right) \frac{v(\alpha(-|z|))}{v\left(\alpha\left(-|z|^{2}\right)\right)} \leq\|h\|_{u}
$$

since

$$
\operatorname{Im} \alpha(-|z|)=\frac{1-|z|}{1+|z|} \leq \frac{1-|z|^{2}}{1+|z|^{2}}=\operatorname{Im} \alpha\left(-|z|^{2}\right) .
$$

Hence $S h \in X$.

Conversely, if $h \in X$ then $h(z)=k\left(z^{2}\right)$ for some holomorphic function $k: \mathrm{D} \rightarrow \mathrm{C}$. Hence $S^{-1} h=k$. We have, with $z=z_{0}^{2}$

$$
\begin{aligned}
\left|\left(S^{-1} h\right)(z)\right| u(z) & =\left|k\left(z_{0}^{2}\right)\right| u(z)=\left|h\left(z_{0}\right)\right| u\left(z_{0}\right) \frac{u(z)}{u\left(z_{0}\right)} \\
& \leq\|h\|_{u} \frac{v\left(\alpha\left(-\left|z_{0}\right|^{2}\right)\right.}{v\left(\alpha\left(-\left|z_{0}\right|\right)\right)} \\
& \leq\|h\|_{u} c\left(\frac{1-\left|z_{0}\right|^{2}}{1+\left|z_{0}\right|^{2}}\right)^{\beta}\left(\frac{1+\left|z_{0}\right|}{1-\left|z_{0}\right|}\right)^{\beta} \\
& \leq c 2^{2 \beta}\|h\|_{u} .
\end{aligned}
$$

Hence $d(X, H u(\mathrm{D})) \leq c 2^{2 \beta}$. This together with (3.4) implies the proposition.

We show next that $U_{v}$ is complemented in $H v(\mathrm{G})$.

Proposition 3.2. There is a constant $d$ which depends only on $\beta$ and $c$ such that

$$
\lambda\left(U_{v}, H v(\mathrm{G})\right) \leq d .
$$

Proof. Let $w_{k}, k=1, \ldots, \beta$, be the zeros of $w^{2 \beta}+1$ in G, i.e. $w_{k}=$ $\exp \left(i(2 k-1)(2 \beta)^{-1} \pi\right)$. Then $\operatorname{Im} w_{k} \geq \sin \left((2 \beta)^{-1} \pi\right)$ for all $k$. Let $O_{k}$ be the open disc with center $w_{k}$ and radius

$$
r:=\min \left(\frac{\sin (\pi /(2 \beta))}{2}, \min \left\{\frac{\left|w_{k}-w_{j}\right|}{2}: k, j=1, \ldots, \beta, k \neq j\right\}\right) .
$$


Then the $O_{k}$ are mutually disjoint. Finally let $\delta=\inf \left\{\left|w^{2 \beta}+1\right|: w \in\right.$ $\left.\mathrm{G} \backslash \bigcup_{k=1}^{\beta} O_{k}\right\}$. Put

$$
V=\left\{f \in H v(\mathrm{G}): f\left(w_{k}\right)=0, k=1, \ldots, \beta\right\} .
$$

Then $V$ is $\beta$-codimensional in $H v(\mathrm{G})$ and the codimension of $U_{v} \cap V$ in $U_{v}$ is $\leq \beta$. For $f \in V$ put

$$
(T f)(w)=\frac{1}{w^{2 \beta}+1}\left(f(w)+f\left(-\frac{1}{w}\right)\right) .
$$

Then $T f$ is holomorphic. This follows from the fact that $-1 / w_{k}$ is a zero of $w^{2 \beta}+1$ in $\mathrm{G}$ for all $k$, too. We claim $T f \in U_{v}$. Indeed, (3.1) implies

$$
\frac{v(w)}{v(-1 / w)} \leq \begin{cases}1, & |w| \leq 1 \\ c|w|^{2 \beta}, & |w| \geq 1\end{cases}
$$

Consider $w \in \mathrm{G}$ with $|w| \geq 2$. Then, in view of (3.5),

$$
\begin{aligned}
|(T f)(w)| v(w) & \leq \frac{1}{2^{2 \beta}-1}\|f\|_{v}+\frac{v(w)}{\left|w^{2 \beta}+1\right| v(-1 / w)}\left|f\left(\frac{-1}{w}\right)\right| v\left(\frac{-1}{w}\right) \\
& \leq \frac{2^{2 \beta} c+1}{2^{2 \beta}-1}\|f\|_{v} .
\end{aligned}
$$

Next, let $w \in \mathrm{G} \backslash \bigcup_{k=1}^{\beta} O_{k}$ such that $|w| \leq 2$. Then $|(T f)(w)| v(w) \leq \delta^{-1}(1+$ $\left.c 2^{2 \beta}\right)\|f\|_{v}$. (Again, we used (3.5).) Finally, let $w \in \bigcup_{k=1}^{\beta} O_{k}$, say $w \in O_{j}$. Since $T f$ is holomorphic, by the maximum principle, there is $w_{0} \in \partial O_{j}$ with $\mid(T f) w)|\leq|(T f)\left(w_{0}\right) \mid$. Hence, with (3.1),

$$
\begin{aligned}
|(T f)(w)| v(w) & \leq \frac{v(w)}{v\left(w_{0}\right)}\left|(T f)\left(w_{0}\right)\right| v\left(w_{0}\right) \\
& \leq \frac{v\left(\operatorname{Im} w_{j}+r\right)}{v\left(\operatorname{Im} w_{j}-r\right)}\left|(T f)\left(w_{0}\right)\right| v\left(w_{0}\right) \\
& \leq \frac{1}{\delta}\left(1+2^{2 \beta} c\right) c\left(\frac{\operatorname{Im} w_{j}+r}{\operatorname{Im} w_{j}-r}\right)^{\beta}\|f\|_{v} \\
& \leq \frac{1}{\delta}\left(1+2^{2 \beta} c\right) c\left(\frac{\frac{3}{2} \sin \left(\frac{\pi}{2 \beta}\right)}{\frac{1}{2} \sin \left(\frac{\pi}{2 \beta}\right)}\right)^{\beta}\|f\|_{v} \\
& =\frac{3^{\beta}}{\delta} c\left(1+2^{2 \beta} c\right)\|f\|_{v} .
\end{aligned}
$$


Thus

$$
\|T f\|_{v} \leq \max \left(\frac{2^{2 \beta} c+1}{2^{2 \beta}-1}, \frac{3^{\beta}}{\delta} c\left(1+2^{2 \beta} c\right)\right)\|f\|_{v} .
$$

Clearly, $w^{2 \beta}(T f)(w)=(T f)(-1 / w)$ which shows $T f \in U_{v}$. If $f \in U_{v} \cap V$ then $T f=f$.

Let $Q_{1}: U_{v} \rightarrow U_{v} \cap V$ and $Q_{2}: H v(\mathrm{G}) \rightarrow V$ be projections with $\left\|Q_{j}\right\| \leq \beta, j=1,2$. Then $Q_{1} T Q_{2}$ is a projection from $H v(\mathrm{G})$ onto $U_{v} \cap V$ and $\operatorname{dim}\left(i d-Q_{1} T Q_{2}\right) U_{v} \leq \beta$. Hence we find a projection $Q_{3}: H v(\mathrm{G}) \rightarrow$ $\left(i d-Q_{1} T Q_{2}\right) U_{v}$ with $\left\|Q_{3}\right\| \leq \beta$. Finally put

$$
P=Q_{3}\left(i d-Q_{1} T Q_{2}\right)+Q_{1} T Q_{2}
$$

Then $P$ is a projection from $H v(\mathrm{G})$ onto $U_{v}$ with $\|P\| \leq \beta\left(1+\beta^{2}\|T\|\right)+$ $\beta^{2}\|T\|$. This together with (3.6) completes the proof of Proposition 3.2.

\section{Proof of Theorem 1.2}

Consider a standard weight $v$ on G satisfying $(\star)$. Put

$$
u_{n}(z)=v\left(n \frac{1-|z|}{1+|z|} i\right), \quad z \in \mathrm{D}, \quad n \in \mathrm{N},
$$

and assume

$$
a_{n}:=\sup _{j \in \mathrm{N}} \frac{u_{n}\left(1-2^{-j}\right)}{u_{n}\left(1-2^{-j-1}\right)}<\infty \quad \text { for each } n .
$$

Fix integers $0=m_{n, 0}<m_{n, 1}<m_{n, 2}<\cdots$ with

$$
\frac{1}{2 a_{n}} \leq \frac{u_{n}\left(1-2^{-m_{n, k+1}}\right)}{u_{n}\left(1-2^{-m_{n, k}}\right)} \leq \frac{1}{2} .
$$

Then we have

LEMMA 4.1.

(i) $v$ satisfies $(\star)$ if and only if $\sup _{n} a_{n}<\infty$.

(ii) Let $v$ satisfy $(\star)$.

Then $v$ also satisfies $(\star \star)$ if and only if $\sup _{n} \sup _{k}\left(m_{n, k}-m_{n, k-1}\right)<\infty$.

Proof. (i) Fix $n \in \mathrm{N}$. Let $m \in \mathrm{N} \cup\{0\}$ such that $2^{m} \leq n \leq 2^{m+1}$. Then we have

$$
\frac{u_{n}\left(1-2^{-j}\right)}{u_{n}\left(1-2^{-j-1}\right)}=\frac{v\left(n\left(2^{j+1}-1\right)^{-1} i\right)}{v\left(n\left(2^{j+2}-1\right)^{-1} i\right)} \leq \frac{v\left(2^{m-j+1} i\right)}{v\left(2^{m-j-2} i\right)}
$$


and

$$
\frac{u_{n}\left(1-2^{-j+1}\right)}{u_{n}\left(1-2^{-j-2}\right)}=\frac{v\left(n\left(2^{j}-1\right)^{-1} i\right)}{v\left(n\left(2^{j+3}-1\right)^{-1} i\right)} \geq \frac{v\left(2^{m-j} i\right)}{v\left(2^{m-j-1} i\right)}
$$

From this we infer (i).

(ii) Assume $(\star \star)$. Then there is $j \in \mathrm{N}$ with

$$
b:=\sup _{k \in Z} \frac{v\left(2^{k} i\right)}{v\left(2^{k+j} i\right)}<1 .
$$

We can assume $b \leq 1 / 2$, otherwise take $l j$ instead of $j$ for suitable $l \in \mathrm{N}$. Hence if $n \in \mathrm{N}$ and $2^{m} \leq n \leq 2^{m+1}$ then

$$
\frac{u_{n}\left(1-2^{-l-j-2}\right)}{u_{n}\left(1-2^{-l}\right)}=\frac{v\left(n\left(2^{l+j+3}-1\right)^{-1} i\right)}{v\left(n\left(2^{l+1}-1\right)^{-1} i\right)} \leq \frac{v\left(2^{m-l-1-j} i\right)}{v\left(2^{m-l-1} i\right)} \leq b \leq \frac{1}{2}
$$

for all $l \in \mathrm{N}$. From this we obtain $\sup _{k}\left(m_{n, k}-m_{n, k-1}\right) \leq j+2$.

Now assume $\sup _{n} \sup _{k}\left(m_{n, k}-m_{n, k-1}\right)<\infty$. A simple calculation shows that there is $j \in \mathrm{N}$ with

$$
\frac{u_{n}\left(1-2^{-l-j}\right)}{u_{n}\left(1-2^{-l}\right)} \leq \frac{1}{2} \quad \text { for all } \quad l, n \in \mathrm{N} .
$$

Hence

$$
\sup _{l, n} \frac{v\left(\frac{n}{2^{l+j+1}-1} i\right)}{v\left(\frac{n}{2^{l+1}-1} i\right)} \leq \frac{1}{2}
$$

and we easily infer from this condition $(\star \star)$.

Conclusion of The Proof of TheOrem 1.2. Let $H v(\mathrm{G})$ be isomorphic to $l_{\infty}$. Consider the standard weights $\tilde{v}_{n}(w)=v(n w), n \in \mathrm{N}$. Define $T_{n}$ : $H v(\mathrm{G}) \rightarrow H \tilde{v}_{n}(\mathrm{G})$ by $\left(T_{n} f\right)(w)=f(n w), w \in \mathrm{G}$. The $T_{n}$ are ontoisometries. $(\star)$ implies

$$
\sup _{n \in \mathrm{N}} \sup _{k \in \mathrm{Z}} \frac{\tilde{v}_{n}\left(2^{k+1} i\right)}{\tilde{v}_{n}\left(2^{k} i\right)}<\infty .
$$

Hence, by Lemma 1.6 there are constants $c, \beta>0$ with

$$
\frac{\tilde{v}_{n}(t i)}{\tilde{v}_{n}(s i)} \leq c\left(\frac{t}{s}\right)^{\beta} \quad \text { whenever } 0<s \leq t \text { for all } n \in \mathrm{N} .
$$

Consider the spaces $U_{\tilde{v}_{n}} \subset H \tilde{v}_{n}(\mathrm{G})$. According to Proposition 3.1 and Proposition 3.2 we have

$$
\sup _{n} \lambda\left(U_{\tilde{v}_{n}}, H \tilde{v}_{n}(\mathrm{G})\right)<\infty \quad \text { and } \quad \sup _{n} d\left(U_{\tilde{v}_{n}}, H u_{n}(\mathrm{D})\right)<\infty .
$$


Since $d\left(H \tilde{v}_{n}(\mathrm{G}), l_{\infty}\right)=d\left(H v(\mathrm{G}), l_{\infty}\right)$ we conclude $\sup _{n} \lambda\left(U_{\tilde{v}_{n}}\right)<\infty$ and hence $\sup _{n} \lambda\left(H u_{n}(\mathrm{D})\right)<\infty$. Proposition 2.5 then $\operatorname{shows}_{\sup _{k, n}}\left(m_{n, k}-\right.$ $\left.m_{n, k-1}\right)<\infty$. By Lemma 4.1, $v$ satisfies $(\star \star)$.

Now assume $(\star \star)$. With Lemma 4.1 and Proposition 2.5 we see that $\sup _{n} \lambda\left(H u_{n}(\mathrm{D})\right)<\infty$. Hence $\left(\sum_{n} \oplus H u_{n}(\mathrm{D})\right)_{(\infty)}$ is isomorphic to a complemented subspace of $l_{\infty}$. In view of Corollary $1.5 H v(\mathrm{G})$ is isomorphic to a complemented subspace of $l_{\infty}$. Hence $d\left(H v(\mathrm{G}), l_{\infty}\right)<\infty$ (see [9], Theorem 2.a.7).

It is known that $H v_{0}(\mathrm{G})^{* *}$ is isomorphic to $H v(\mathrm{G})$ ([2], [5]). Hence if $H v_{0}(\mathrm{G})$ is isomorphic to $c_{0}$ then $H v(\mathrm{G})$ is isomorphic to $l_{\infty}$.

Conversely, if $H v(\mathrm{G})$ is isomorphic to $l_{\infty}$ then $H v_{0}(\mathrm{G})$ is a $\mathscr{L}_{\infty}-\operatorname{space}([8])$. $H v_{0}(\mathrm{G})$ is always isomorphic to a subspace of $c_{0}$ ([3]). By [6] this means that $H v_{0}(\mathrm{G})$ is isomorphic to $c_{0}$.

CONCLUDING REMARKS. It is known that, for any radial decreasing weight $u$ on $\mathrm{D}$, the space $H u(\mathrm{D})$ is either isomorphic to $l_{\infty}$ or to $H_{\infty}$, the space of all bounded holomorphic functions on D (with the sup-norm), see [11]. It is very likely that, for a standard weight $v$ on G which satisfies $(\star)$ but not $(\star \star)$, the space $H v(\mathrm{G})$ is isomorphic to $H_{\infty}$, too. Even without condition ( $\star$ ) there might be only two isomorphism classes for $H v(\mathrm{G})$, namely $l_{\infty}$ and $H_{\infty}$. However, we mention again that in any case $v$ must satisfy $v(t i) \leq e^{b t}, t>0$, for some constant $b \in \mathrm{R}$. (This is always the case if $(\star)$ holds.) Otherwise, according to [15], $H v(\mathrm{G})=\{0\}$.

\section{REFERENCES}

1. Agethen, S., Weighted Banach spaces of holomorphic functions on the upper half-plane, Bull. Soc. Roy. Sci. Liège 73 (2004), 69-80.

2. Bierstedt, K. D., and Summers, W. H., Biduals of weighted Banach spaces of analytic functions, J. Austral. Math. Soc. (A) 54 (1993), 70-79.

3. Bonet, J., and Wolf, E., A note on weighted Banach spaces of holomorphic functions, Arch. Math. (Basel) 81 (2003), 650-654.

4. Domanski, P., and Lindström, M., Sets of interpolation and sampling for weighted Banach spaces of holomorphic functions, Ann. Polon. Math. 79 (2002), 233-264.

5. Holtmanns, S., Operator representation and biduals of weighted function spaces, $\mathrm{PhD}$ thesis, Inst. for Math., Univ. of Paderborn 2000.

6. Johnson, W. B., and Zippin, M., On subspaces of quotients of $\left(\sum G_{n}\right)_{l_{p}}$ and $\left(\sum G_{n}\right)_{c_{0}}$, Israel J. Math. 13 (1972), 311-316.

7. Kadec, M. $\breve{\text { I., }}$ and Snobar, M. G., Certain functionals on the Minkowski compactum, Mat. Zametki 10 (1971), 453-458, English translation: Math. Notes 10 (1971), 695-696.

8. Lindenstrauss, J., and Rosenthal, H. P., The $\mathscr{L}_{p}$ spaces, Israel J. Math. 7 (1969), 325-349.

9. Lindenstrauss, J., and Tzafriri, L., Classical Banach Spaces I, Ergebn. Math. Grenzgeb. 92, Springer, Berlin 1977.

10. Lusky, W., On weighted spaces of harmonic and holomorphic functions, J. London Math. Soc. (2) 59 (1995), 309-320. 
11. Lusky, W., On the isomorphism classes of weighted spaces of harmonic and holomorphic functions, Studia Math. 175 (2006), 19-45.

12. Shields, A. L., and Williams, D. L., Bounded projections, duality, and multipliers in spaces of analytic functions, Trans. Amer. Math. Soc. 162 (1971), 287-302.

13. Shields, A. L., and Williams, D. L., Bounded projections, duality, and multipliers in spaces of harmonic functions, J. Reine Angew. Math. 299/300 (1978), 256-279.

14. Shields, A. L., and Williams, D. L., Bounded projections and the growth of harmonic conjugates in the unit disc, Michigan Math. J. 29 (1982), 3-25.

15. Stanev, M. A., Weighted Banach spaces of holomorphic functions in the upper half plane, preprint 1999, arXiv: math.FA/9911082.

16. Torchinsky, A., Real-Variable Methods in Harmonic Analysis, Pure Appl. Math. 123, Academic Press, Orlando, FL 1986.

17. Wojtaszczyk, P., Banach Spaces for Analysts, Cambridge Studies Adv. Math. 25, Cambridge Univ. Press, Cambridge 1991.

DEPARTMENT OF MATHENATICS

FACULTY OF SCIENCE

UNIVERSITY OF KURDISTAN

PASDARAN AVE.

SANANDAJ

POSTAL CODE: 66177-15175

IRAN

E-mail: M.Ardalani@uok.ac.ir
INSTITUTE FOR MATHEMATICS UNIVERSITY OF PADERBORN

WARBURGER STR. 100

D-33098 PADERBORN

GERMANY

E-mail: lusky@math.upb.de 